\title{
Historia y física: a propósito de las críticas de Larcher a Voltaire*
}

\author{
History and Physics: \\ Regarding Larcher's critique to Voltaire
}

\author{
ADRIÁN RATTO \\ Universidad de Buenos Aires
}

Recibido: 08-IX-2014 Aprobado definitivamente: 12-X-2014

RESUMEN

El objetivo de este trabajo es evaluar el alcance del programa de Voltaire para acercar la historia a la nueva ciencia, presentado en sus Nouvelles considerations sur l'histoire. A la luz de las críticas que Larcher le dirige a propósito de su Philosophie de l'histoire y su respuesta en La défense de mon oncle, se demuestra que existen diferentes criterios epistemológicos en los trabajos históricos e historiográficos de Voltaire y que la articulación entre los mismos es problemática. Esto pone de relieve los límites de su proyecto historiográfico.

\section{PALABRAS CLAVE \\ HISTORIA-CIENCIA-ILUSTRACIÓN-VOLTAIRE-LARCHER}

\section{ABSTRACT}

The aim of this paper is to examine the extent of Voltaire's program, in order to bring the history to the new science, presented in his Nouvelles considerations sur l'histoire. In

\footnotetext{
* Este trabajo se ha realizado en el marco del proyecto "Philosophy of History and Globalisation of Knowledge. Cultural Bridges Between Europe and Latin America”(F7-PEOPLE-2013-IRSES: 612644), financiado por la Comisión Europea.
}

(C) Contrastes. Revista Internacional de Filosofia, vol. XX No 2 (2015), pp. 297-312. ISSN: 1136-4076 Licenciatura de Filosofía, Universidad de Málaga, Facultad de Filosofía y Letras Campus de Teatinos, E-29071 Málaga (España) 
the light of the critiques that Larcher gives to him about his Philosophie de l'histoire and its response in La défense de mon oncle, it is shown that there are different epistemological criteria in historical and historiographical works of Voltaire and that the joint between them is problematic. This highlights the boundaries of his historiographical project.

KEYWORDS

HISTORY-SCIENCE-ENLIGHTENMENT-VOLTAIRE-LARCHER

\section{INTRODUCCIÓN}

E1 20 de marzo de 1767 aparecía, sin nombre de autor, un Supplément à la Philosophie de l'histoire de feu M. l'Abbé Bazin (Amsterdam: Changuion). El propósito de la obra era, como adelanta en el prefacio Pierre-Henri Larcher, autor del Supplément, sacar a la luz los errores cometidos por Voltaire en su Philosophie de l'histoire (1765). ${ }^{1}$ Los trabajos históricos del autor de la Henriade habían ya recibido críticas como, por ejemplo, las del jesuita Claude-François Nonnotte en Les erreurs de Voltaire (1762) o las de Louis Viret en su Réponse à la Philosophie de l'histoire (1767). ${ }^{2}$ En esos casos el blanco de las críticas era principalmente el tratamiento que Voltaire daba a la religión. Larcher, en cambio, cuestionaba la autoridad del francés como historiador. Voltaire preparó inmediatamente su defensa a través de La défense de mon oncle, publicada ese mismo año. ${ }^{3}$

Sorprendentemente, Voltaire, que poco tiempo antes en la entrada «HisTOIRE» del volumen octavo de la Encyclopédie (1765) había expresado su deseo de acercar la historia a los nuevos métodos de la ciencia experimental, evitaba aparentemente en su respuesta a Larcher el terreno de la «historia científica», limitándose a desacreditar a su adversario a través de la burla y la crítica personal. El propósito de este trabajo es evaluar el alcance de la respuesta de Voltaire a Larcher, un tema que aún no ha sido suficientemente analizado por los especialistas, quienes en general circunscriben las críticas de Voltaire a Larcher al plano biográfico. ${ }^{4}$ Se intentará demostrar que la respuesta de Voltaire

1 Cf. P. Larcher, Supplément à la Philosophie de l'histoire de feu M. l'Abbé Bazin, nécessaire à ceux qui veulent lire cet ouvrage avec fruit. Amsterdam: Changuion, 1767, pp. 35, 36.

2 Se podría mencionar también Ch.-F. Schmidt, La philosophie de l'histoire, par feu l'abbé Bazin, critiquée par Ch.-F. Schmidt. Jacobaer: Leipsig, 1766 y J.-G. Clémence, Défense des livres de l'Ancien Testament, contre l'écrit de Voltaire intitulé: La Philosophie de l'histoire. Paris: Pillot, 1768.

3 Larcher respondió ese mismo año (1767) a través de una Réponse à la Défense de mon oncle (Amsterdam: Changuion) y dos años después (1769) publicó una segunda edición aumentada del Supplément.

4 Se han dedicado hasta el momento pocos trabajos al tema. Al respecto, véase J.-M. Moreaux, «Voltaire et Larcher, ou le faux “mazarinier”», Revue d'Histoire littéraire de la France, 
a Larcher desborda el plano personal, así como también la coyuntura ideológico-política (la lucha entre Voltaire y les anti-philosophes), inscribiéndose en el plano de la metodología y la filosofía de la historia. Esto, por otra parte, nos permitirá esclarecer algunos aspectos de la problemática articulación entre ciencia e historia en la obra del francés.

La estructura del trabajo es la siguiente: en primer lugar (I), nos detendremos en algunas consideraciones generales acerca del interés de Voltaire en acercar la historia al nuevo método científico, representado paradigmáticamente por la física de Newton; luego (II), revisaremos detalladamente las principales críticas de Larcher a Voltaire y las respuestas esgrimidas por éste en La défense de mon oncle; finalmente (III), analizaremos la articulación entre los diferentes criterios utilizados por Voltaire en sus críticas a Larcher, con el propósito de evaluar, a la luz de ese análisis, el alcance y los límites del proyecto de Voltaire de acercar la historia a la nueva física.

\section{HISTORIA Y FÍSICA}

Los Eléments de la philosophie de Newton (1738) conocieron veintiséis ediciones entre 1738 y 1785. La Philosophice Naturalis Principia Mathematica había sido publicada en Londres en $1687,{ }^{5}$ pero los franceses eran, en 1738 , aún devotos de la física de los torbellinos de Descartes. Si bien Voltaire no fue el único responsable de la introducción de Newton en Francia, sus Eléments alteraron el panorama intelectual francés. El fin del autor de la Henriade, que había entrado en contacto con las obras de Newton, Locke y Bacon, entre otros, durante su exilio en Inglaterra, no era sólo modificar la interpretación

74, nº 4 (1974), pp. 600-626; R. Mcginnis, «L'histoire prostituée. Voltaire contre Larcher, et contre lui-même», The Romanic Review, 88, n 2 (1997), pp. 229-240. Acerca de los críticos y las disputas de Voltaire en general, véase U. Kölvig y Ch. Mervaud (eds.), Voltaire et ses combats. Oxford/Paris: Voltaire Foundation, 1994 y O. Ferret, «Voltaire: pamphlets and polemic», en N. Cronk (ed.), The Cambridge Companion to Voltaire. Cambridge: Cambridge University Press, 2009, pp. 167-179. Para un panorama acerca del estado actual de los estudios sobre los trabajos históricos e historiográficos de Voltaire, véase J.-M. Moureaux, «Voltaire historien: un chantier qui s'ouvre», Revue d'histoire littéraire de la France, 101 (2002), pp. 227-261; véase también el número 12 (2012) de la Revue Voltaire, que contiene el dossier «Voltaire historien», dirigido por Myrtille Méricam-Bourdet.

5 La obra fue publicada originalmente en latín. La primera traducción al francés fue realizada por la marquesa Émilie du Châtelet. Émilie comenzó la traducción hacia 1736 durante la estadía de Voltaire en Cirey. El libro fue publicado en 1759, luego de la muerte de la marquesa. Véase R. Pomeau, «Voltaire et Mme. du Châtelet à Cirey: amour et travail», Studies on Voltaire and the Eighteenth Century, 11 (2001), pp. 9-15 [el artículo forma parte del dossier «Cirey dans la vie intellectuelle: La réception de Newton en France», dirigido por François de Gandt]. 
de sus compatriotas acerca del mundo físico, sino también producir una revolución desde el punto de vista de la metodología del conocimiento. En este marco se inscribe su intención de acercar la historia a los métodos de la física experimental. «Quizá pronto ocurra en la manera de escribir historia, lo que ha sucedido en la física. Los nuevos descubrimientos han vuelto obsoletos los antiguos sistemas», dice al comienzo de sus Nouvelles considerations sur l'histoire (1744), donde presenta un programa para la renovación de la historia. Inmediatamente agrega: «se querrá conocer al género humano con el mismo rigor alcanzado en la filosofía natural». ${ }^{6}$ Paolo Casini considera que existe un «paralelismo metodológico» entre los Eléments de la philosophie de Newton (1738) y los trabajos históricos de Voltaire. ${ }^{7}$ Ahora bien, ¿qué significaba para Voltaire acercar la historia a la ciencia?

Se exige a los historiadores modernos más detalles, hechos registrados, referencias bibliográficas, datos precisos, más atención a los usos, las leyes, las costumbres, el comercio, las finanzas, la agricultura, la demografía. En historia, como en matemáticas y física, el campo de trabajo se ha expandido notablemente. ${ }^{8}$

Así se expresa en la ya mencionada entrada «HistoIRE» de la Encyclopédie. En resumen, acercar la historia a la nueva ciencia significaba dos cosas para Voltaire: por un lado (i), someter el relato histórico a un control riguroso; por el otro (ii), ampliar los límites espacio-temporales y los objetos de la narración, evitando las «particularizaciones» de la historia, ya sean eclesíasticas o políticas.

Con respecto al primer criterio (i), Voltaire busca separar las «fábulas» de la «historia». La historia, dice, «es el relato de hechos considerados verdaderos, en cambio, la fábula es el relato de hechos considerados falsos». ${ }^{9} \mathrm{El}$ francés propone escribir historia de manera rigurosa, apoyando el relato en

6 Voltaire, Nouvelles considérations sur l'histoire, en Voltaire, CEuvres historiques, ed. R. Pomeau. Paris: Gallimard, 1957, p. 46. La intención de acercar la historia a la fisica también aparece en la entrada «HisTOIRE» (1765), redactada por Voltaire para la Encyclopédie y en Le pyrrhonisme de l'histoire (1769). Las traducciones de los pasajes de las obras de Voltaire son nuestras.

7 Cf. P. Casini, «Progrès de la raison et progrès des sciences chez les encyclopédistes», en L'histoire au dix-huitième siècle. Aix-en-Provence: EDISUD, 1980, pp. 121-122. La idea de un paralelismo entre historia y física en la obra de Voltaire también está presente en el estudio preliminar de René Pomeau a su edición crítica del Essai sur les mœurs et l'esprit des nations [Paris: Garnier, 1964, vol. 1, p. XXIX. En adelante Essai].

8 Voltaire, «HistorRe», en D. Diderot y J. D’Alembert (eds.), Encyclopédie ou Dictionnaire raisonné des sciences, des arts et des métiers. Paris: Briasson/David/Le Breton/ Durand, 1765, vol. 8, p. 225.

9 Ibid., p. 220. 
hechos, testimonios y registros públicos, y tomando distancia respecto de las historias romanesques y las fábulas. Al final del Essai sur les mœurs et l'esprit des nations (1756), señala que «un lector inteligente comprenderá fácilmente que no debe dar crédito más que a los acontecimientos que tienen cierta verosimilitud, y mirar con desdén las fábulas con las que el fanatismo, el espíritu novelesco y la credulidad han desfigurado en todos los tiempos la historia». ${ }^{10}$ Esto lo llevó a alejarse de aquellos que, como por ejemplo Géraud de Cordemoy (1626-1684), habían afirmado que el historiador tenía que ocuparse de la belleza y la claridad del estilo antes que de la veracidad de la información acerca del pasado, ${ }^{11}$ pero también de aquellos que, como Claude Fleury en su Histoire ecclésiastique (1691) o Charles Rollin en Histoire romaine depuis la fondation de Rome jusqu'à la bataille d'Actium (1738-1748), confundían la historia y la religión o retomaban en su época viejos y absurdos relatos.

Ahora bien, ¿cuál es el criterio para diferenciar la «historia verdadera» de las «fábulas», según el francés? La «verosimilitud», responde: «todo conocimiento que no alcanza una demostración matemática, no se apoya más que en la probabilidad. No hay otro tipo de conocimiento en historia», afirma. ${ }^{12} \mathrm{De}$ esta manera, se alejaba tanto de los cartesianos, que en el siglo XVII habían negado un lugar a la historia en el «árbol del conocimiento», tomando como modelo la matemática, y de los libertinos como Pierre Bayle o François de La Mothe Le Vayer que en la misma época ponían de relieve las absurdidades y contradicciones que recorrían los relatos históricos vigentes y se mantenían escépticos respecto del estatus epistémico de la historia. ${ }^{13}$ "Yo no busco ni un pirronismo exagerado, ni una credulidad ridícula», ${ }^{14}$ señala Voltaire, refiriéndose al conocimiento histórico, en Le pyrrhonisme de l'histoire (1769).

Pero, ¿cómo se determina la probabilidad de un suceso? El autor del Essai menciona dos parámetros, a saber, (a) la existencia de pruebas que apoyen el relato histórico y (b) la adecuación del suceso histórico al «orden de la

10 Essai, vol. 2, p. 800.

11 Cf. G. de Cordemoy, Ce qu'on doit observer en écrivant l'Histoire, en Divers traitez (sic) de métaphysique, d'histoire, et de politique. Paris: Coignard, 1691, pp. 68, 69.

12 Voltaire, «HistorRE», ed. cit., p. 223. Asimismo, en el Essai dice: «no admitamos en física sino aquello que ha sido probado y en historia aquello que tiene un alto grado de probabilidad», en Essai, p. 203.

13 Acerca de la historia en el siglo XVII, véase B. Guion, «Comment écrire l'histoire: l'ars historica à l'âge classique», Dix-septième siècle, 246 (2010), pp. 9-25. Véase también, de la misma autora, Du bon usage de l'histoire: histoire, morale et politique à l'âge classique. Paris: Honoré Champion, 2008.

14 Voltaire, Le pyrronhisme de l'histoire, en Voltaire, Euvres complètes, ed. L. Moland. Paris: Garnier, 1879, t. 27, p. 235. 
naturaleza»». ${ }^{15}$ Con respecto a las pruebas (a), existen, según Voltaire, diferentes tipos: testimonios directos, archivos y monumentos. Así, por ejemplo, «la existencia de un gran número de testimonios, provenientes de personas de diferentes naciones y la ausencia de objeciones» acerca de la numerosa población de China, es suficiente para que Voltaire califique ese dato como cierto. ${ }^{16} \mathrm{En}$ el mismo sentido, en el prefacio a la Histoire de Charles XII (1731), uno de sus primeros trabajos históricos, señala que la obra se apoya en «hechos probados por testimonios visuales irreprochables» y que eso diferencia su trabajo de las vidas de Carlos XII que habían aparecido hasta ese momento ${ }^{17}$ En cuanto a los otros tipos de pruebas, al final del Essai aconseja no dar crédito más que a «los eventos atestados por los registros públicos» ${ }^{18}$ y en el artículo «HIstoIRE», al hablar del Medioevo, dice que «para penetrar en el oscuro medioevo es necesario el auxilio de archivos». ${ }^{19}$ Finalmente, además de los archivos y los testimonios directos, Voltaire considera que los monumentos también pueden servir como prueba: «para conocer con certeza alguna cosa de la historia antigua, sólo existe un medio, buscar algún monumento de la época» ${ }^{20}$ En el Essai señala, no obstante, que los monumentos sólo sirven como prueba «cuando son mencionados por autores renombrados». La mayoría de los monumentos y medallas, agrega, «no prueban más que errores consagrados». ${ }^{21}$

En cuanto al segundo parámetro (b), Voltaire sostiene que «no se debe creer en aquello que es contrario al curso ordinario de la naturaleza».22 Así, por ejemplo, afirma que es necesario aceptar como verdaderas las aventuras de Carlos XII en la medida en que existen numerosos testimonios al respecto y estos «no son contrarios a las leyes de la naturaleza»». ${ }^{23}$ La misma norma es utilizada, por ejemplo, en el capítulo dedicado a los griegos en su Philosophie de l'histoire, para desacreditar los relatos históricos acerca de esa nación.

15 Cf. Voltaire, «HistorRE», ed. cit., p. 221.

16 Ibid., p. 224.

17 Voltaire, Histoire de Charles XII, en Voltaire, Euvres historiques, ed. cit., p. 54. Para una interpretación diferente, véase J. Brumfitt, Voltaire Historian. Oxford: Oxford University Press, 1958, p. 26: «la Histoire de Charles XII tiene aún muchas afinidades con la tradición historiográfica del siglo XVII».

18 Essai, p. 802.

19 Voltaire, «HISTOIRE», ed. cit., p. 223.

20 Ibid., p. 221.

21 Essai, p. 801.

22 Voltaire, «HistoIRE», ed. cit., p. 224.

23 Ibid. Sobre la problemática idea de «naturaleza» en el siècle des Lumières, véase J. Ehrard, L'idée de nature en France dans la première moitié du XVIII siècle. Paris: Albin Michel, 1963. 
Sólo se puede creer, dice, en aquellos relatos que «son verosímiles y que no contradicen el orden de la naturaleza».24

Con respecto al segundo criterio (ii) que se exige para poner la historia a la altura de la nueva ciencia, Voltaire señala que una historia a la altura de los tiempos de la Encyclopédie debe evitar las «particularizaciones» y ser, por lo tanto, una historia general, «una historia de los hombres». ${ }^{25} \mathrm{La}$ «historia moderna» no podía reducirse, según el francés, a la historia de «tres o cuatro naciones», como ocurría en los Discours sur l'histoire universelle (1681) de Jacques Bénigne Bossuet o a la superficial narración de la vida de las cortes y los reyes. ${ }^{26}$ Con este programa Voltaire se interesa en el Essai y en otros trabajos históricos por la historia de disciplinas y lugares que hasta ese momento no habían logrado atraer en general el interés de los historiadores: «China», «India», las «artes», las «finanzas», las «costumbres», los «(cultos», las «leyes», etc.

Ahora bien, Larcher pertenecía al campo de los eruditos. ${ }^{27}$ Por esa razón, el rigor metodológico y la imparcialidad eran aspectos seriamente considerados en sus trabajos. Resulta llamativa, entonces, la polémica con Voltaire en la medida en que los autores parecen compartir estándares y objetivos. Es necesario, entonces, reconstruir y revisar la polémica para arrojar luces sobre el asunto.

\section{LAS CRÍTICAS DE LARCHER Y LAS RESPUESTAS DE VOLTAIRE}

El jesuita Claude-François Nonnotte (1711-1793) publicó en 1762 Les erreurs de Voltaire, trabajo que conoció quince reediciones (la última en 1823). El libro es una respuesta al Essai sur les mœurs (1756). El lector espera encontrar una larga lista de «errores» históricos, pero los «errores» pertenecen sobre todo al plano religioso. Es decir, el autor sugiere que Voltaire se equivoca principalmente porque se aleja del terreno de la fe. Mientras que las «luces de la Religión perfeccionan la razón», el espíritu filosófico «la extravía», dice Nonnotte en las primeras páginas del libro. ${ }^{28}$ Nonnotte se detiene, así, en temas como el «deísmo» de Voltaire, la «naturaleza del alma», el «pecado

24 Cf. Voltaire, Philosophie de l'histoire, ed. J.-H. Brumfitt, en CEuvres complètes de Voltaire/Complete Works of Voltaire. Oxford: Voltaire Foundation, 1969, vol. 59, p. 173.

25 Cf. Voltaire, Nouvelles considérations sur l'histoire, ed. cit., p. 48.

26 Cf. Voltaire, Supplément à l'Essai sur les moeurs, en Essai, p. 197

27 Acerca de la erudición en el siècle des Lumières, véase Ch. Grell, L'histoire entre érudition et philosophie: étude sur la connaissance historique à l'âge des Lumières. Paris: Presses universitaires de France, 1993.

28 C.-F. Nonnotte, Les erreurs de Voltaire. Besançon: Gauthier Frères, 1818, p. 8. Voltaire responde a través de sus Éclaircissements historiques, publicados en la edición de 1763 del Essai sur les mœurs (Genève: Cramer). Así se desata una larga polémica entre Nonnotte y Voltaire. Al respecto véase R. Pomeau, Voltaire en son temps. Paris: Fayard, 1995, vol. 2, pp. 220 y ss. 
original», etc. El propósito de Louis Viret no es diferente. En la presentación de su Réponse à la Philosophie de l'histoire (1767) declara que su objetivo es defender la religión frente al «despotismo filosófico dominante». ${ }^{29}$ Viret señala que su «plan» es simplemente «responder a las objeciones dirigidas contra la revelación». ${ }^{30}$

El caso de Larcher es diferente. Como bien ha demostrado José-Michel Moreaux, no se puede asociar a Larcher con personajes como Coger, Viret, Riballier, Patouillet o Nonnotte, es decir con los anti-philosophes. ${ }^{31}$ Por el contrario, Larcher fue un filólogo, un reconocido helenista que fue miembro de la Académie des sciences de Dijón y de la Académie des inscriptions et belleslettres de París y que dedicó más de veinte años a traducir a Heródoto. Además, llegó a colaborar con el mismo Buffon en una traducción al francés de las Philosophical Transactions de la Royal Society of London y a integrar el círculo de amistades del barón d'Holbach. De esta manera no es sorprendente que sus críticas a Voltaire en el Supplément no giren en torno a la relación entre filosofía y religión, sino a cuestiones metodológicas y epistemológicas. En efecto, Larcher critica a Voltaire, «colocándose dentro de los límites de la erudición». ${ }^{32}$ Voltaire, dice, «pese a desconocer las lenguas clásicas (...), la historia, pese a ignorar los principios de la crítica, analizó los monumentos de la Antigüedad». Por esta razón, concluye, «no debe sorprendernos verlo superponer un error sobre otro». ${ }^{33}$ "Mi intención es poner en evidencia los plagios, las falsas citas, los errores en la interpretación y los trazos de ignorancia acerca de la historia y la cronología», agrega poco después. ${ }^{34}$ Larcher critica al abbé Bazin - ése es el seudónimo utilizado por Voltaire en su Philosophie de l'histoire - por plagiar los trabajos de autores como Samuel Bochart o Pierre-Daniel Huet, ${ }^{35}$ por citar autores cuyas obras desconoce, como Porfirio o Teodoro, ${ }^{36}$ o por confundirse al traducir o interpretar palabras en lengua extranjera, por ejemplo al escribir

29 Cf. L. Viret, Réponse à la philosophie de l'histoire. Lyon: Duplain, 1767, p. 2.

30 Ibid., p. 6.

31 Cf. J.-M. Moreaux, «Voltaire et Larcher, ou le faux “mazarinier”», ed. cit., p. 601.

32 P. Larcher, op. cit., p. 35. Las traducciones son nuestras.

33 Ibid., p. 35

34 Ibid.

35 Ibid., p. 115

36 Ibid., p. 119. Stéphane Lojkine sostiene que los trabajos históricos de Voltaire, particularmente en el Essai, se apoyan en general en referencias de segunda mano, como los libros Histoire de France, depuis l'établissement de la monarchie françoise dans les Gaules, de Gabriel Daniel o Histoire de France, depuis Faramond jusqu'à maintenant de François de Mézeray, antes que en fuentes originales, cf. S. Lojkine, «La manipulation des sources dans l'Essai sur les mœurs de Voltaire», en B. Binoche y F. Tinland (eds.), Sens du devenir et pensée de l'histoire au temps des lumières. Seyssel: Champ Vallon, 2000, p. 86. 
«muth» en lugar de «mot». ${ }^{37}$ Esto no plantearía un problema, dice Larcher con ironía, si el abbé Bazin se hubiera dedicado a la poesía, pero aquí, agrega, no se trata del «abbé Bazin poeta» sino del «abbé Bazin erudito». ${ }^{38}$

La crítica a la Philosophie de l'histoire significaba, como señala Moreaux, una crítica al «discurso del método» de «Voltaire historiador». ${ }^{39}$ Por esa razón, el francés no tarda en reaccionar. Ese mismo año (1767) publica La défense de mon oncle con el objetivo de defender la Philosophie de l'histoire de las críticas de Larcher. En el libro Voltaire habla a través de la voz de un supuesto «sobrino» del abbé Bazin y no se refiere a Larcher por su nombre real sino, irónicamente, a través del mote «Toxotès». Así, convierte a Larcher en una marioneta y lo acusa de, entre otras cosas, no respetar al género femenino y justificar la sodomía y el incesto. ${ }^{40}$ De esta manera transforma al respetable helenista en un personaje lascivo y caricaturesco, menos interesado en el estudio del pasado que en dar rienda suelta a su propia imaginación.

Si los ataques se detuvieran en este punto, deberíamos acordar con aquellos que consideran que la Défense es un panfleto, un trabajo de coyuntura o un relato en el que la historia se confunde con la ficción. ${ }^{41}$ Pero éstas no son las únicas críticas que Voltaire presenta en el trabajo. Es necesario mencionar, por una parte, una crítica a la erudición de Larcher. En efecto, Voltaire acusa al «pedante» Larcher de no saber hacer otra cosa que «copiar y citar» y de perder de vista lo esencial. ${ }^{42}$ Así, en el capítulo IX, luego de recordar la historia de Sesostris, critica a Larcher por detenerse en detalles y fustiga a «todos los comentaristas» por «ocuparse de la explicación de una palabra inútil y olvidar el fondo de las cosas». ${ }^{43}$ El cuestionamiento al trabajo de los eruditos, es decir, a la acumulación sin sentido de datos y análisis filológicos es una crítica que aparece en diferentes trabajos de Voltaire. ${ }^{44}$ Por otra parte, critica a Larcher por no saber «distinguir la verdad de la mentira, lo probable de lo quimérico». ${ }^{45}$ Por

37 P. Larcher, op. cit, pp. 128, 129.

38 Ibid., p. 129.

39 J.-M. Moreaux, «Introduction», en Voltaire, La défense de mon oncle, ed. crítica de José-Michel Moureaux [en adelante Défense], en Euvres complètes de Voltaire/Complete Works of Voltaire, ed. cit., 1984, t. 64, p. 18. A propósito del interés de Voltaire por el libro de Larcher, véase la correspondencia con D'Alembert y Damilaville de los meses de mayo y junio de 1767, en Voltaire, Correspondance, ed. T. Besterman. Paris: Gallimard, 1983, t. 8.

40 En el capítulo II, por ejemplo, Voltaire acusa a Larcher de ser el detractor del género femenino y en el V y el VI, de haber hecho la apología de la sodomía y el incesto.

41 Cf. J.-M. Moreaux, «Introduction», ed. cit., pp. 5, 128, 129; J. Leigh, Voltaire. A Sense of History. Oxford: Voltaire Foundation, 2004, p. 107.

42 Défense, p. 230.

43 Ibid., p. 214.

44 Cf. Essai, pp. 195 y 883.

45 Défense, p. 230. 
esa razón, por ejemplo, en el capítulo II lo critica por avalar un relato histórico «contrario a la razón». ${ }^{46} \mathrm{El}$ autor del Supplément había explicado, apoyándose en los textos de Heródoto y Jeremías y oponiéndose a lo expresado por Voltaire en la Philosophie de l'histoire, ${ }^{47}$ que en la Antigüedad para cumplir con un ritual religioso las damas de Babilonia debían prostituirse en un templo sagrado por lo menos una vez en su vida. ${ }^{48}$ Voltaire reacciona: «te permito desvariar y proferir injurias a propósito de Camos, Nínive y Assur. Engáñate tanto como quieras acerca de la distancia de Nínive a Babilonia, eso no provoca daño alguno a las damas, por quienes mi tío sentía tanto respeto y a quienes tú ultrajas gravemente. Crees que en los tiempos de Heródoto todas las damas de la inmensa ciudad de Babilonia se prostituían por dinero en el templo. Y lo crees porque Heródoto lo ha dicho».$^{49}$ Quizás estos cuentos hayan entretenido a los griegos, agrega, pero «ningún hombre sensato debe haber dado crédito a los mismos» ${ }^{50}$ Para apoyar la afirmación señala que es inverosímil que, en la sociedad más civilizada de Oriente, hombres tan celosos de sus mujeres las hayan hecho prostituir y afirma que estas cosas repugnan a la naturaleza y la razón. ${ }^{51}$ Hace una evaluación similar con respecto a los temas del incesto, la pederastria y el bestialismo entre las damas de la dinastía de Mendes.

Ahora bien, ¿cómo se articulan estas críticas entre sí? ¿Son compatibles con el programa de Voltaire para acercar la historia a la nueva ciencia? ¿No era también parte de ese programa prestar atención a los detalles y a los archivos? Con el propósito de evaluar el alcance de estas críticas, demostraremos en el

46 Ibid., p. 206.

47 Voltaire, Philosophie de l'histoire, ed. cit., p. 129: «me sorprende que Heródoto haya dicho ante toda Grecia, en su primer libro, que todas las mujeres de Babilonia estaban obligadas por la ley a prostituirse a los extranjeros en el templo de Venus al menos una vez en su vida. Me sorprende aún más que en la actualidad todas las historias hechas para la educación de los jóvenes repitan ese cuento».

48 P. Larcher, op. cit. pp. 111 y ss.

49 Défense, p. 197.

50 Ibid., p. 198. La discusión no se detiene en este punto. Tanto Larcher como Voltaire volverán en numerosas ocasiones sobre el tema en libros y cartas. En 1768, un año después de la publicación de la Défense, Voltaire dice en La Princesse de Babylone: «poned una mordaza al pedante Larcher, que sin saber una palabra de babilonio antiguo, sin haber viajado como yo sobre las riberas del Éufrates y el Tigris, se ha atrevido a sostener que la bella Formosante, hija del rey más importante del mundo, la princesa Aldée y todas las mujeres de esta respetable corte se acostaban por dinero con todos los palafreneros de Asia en el gran templo de Babilonia, para cumplir con un ritual religioso», en Voltaire, La Princesse de Babylone, en Euvres complètes, ed. L. Moland, ed. cit., 1879, t. 21, p. 432. Algunos años después, Larcher contestaría indirectamente a través de su Mémoire sur Vénus (1775). Acerca de la disputa entre Larcher y Voltaire en torno a la prostitución de las damas de Babilonia, véase R. Mcginnis, op. cit.

51 Défense, p. 206. 
siguiente apartado que en los trabajos históriográficos de Voltaire confluyen diferentes estándares y que la articulación entre los mismos es problemática. Esto pondrá de relieve los límites con los que choca el proyecto de Voltaire de acercar la historia a la ciencia experimental.

\section{III. «HisTORIA VERDADERA» E «HiSTORIA DEL ESPÍRITU HUMANO»: VOLTAIRE FRENTE A LOS ERUDITOS}

«¿En qué medida se puede extraer de las aventuras atribuidas a Cariberto, Chilperico y Clotario un conocimiento firme (certaine) y útil (utile)?»> ${ }^{52}$ se pregunta Voltaire en sus Remarques pour servir de supplément à l'Essai sur les mœurs (1763 - un trabajo publicado junto a la $3^{\circ}$ edición del Essai sur les mours et l'esprit des nations. Genève: Cramer). En los trabajos históricos de Voltaire se superponen diferentes criterios metodológicos. La pregunta planteada en las Remarques pone de relieve los dos estándares que guían sus trabajos históricos: la certeza y la utilidad. ${ }^{53}$ Por una lado se trata de elaborar una «historia verdadera» (histoire véritable), una «historia científica» (la science de l'histoire). En ese marco se contrapone la historia a la fábula, se busca escribir acerca del pasado apoyando el relato en diferentes tipos de pruebas (testimonios, archivos y monumentos) y en el ejercicio de la razón, para denunciar la absurdidad de algunos relatos históricos. Por otro lado, Voltaire se interesa por una historia que «hable a la razón», una historia «del espíritu humano» (l'histoire de l'esprit humain). ${ }^{54}$ En Remarques (1763) dice, refiriéndose al tipo de historia que consideraba digna de un espíritu ilustrado como el de Émilie du Châtelet - a quien había dedicado el Essai: «el objeto era la historia del espíritu humano y no los detalles de los sucesos (...); no se trataba, por ejemplo, de averiguar de qué familia era el señor Puiset o el señor Montlhéry, que hicieron la guerra a los reyes de Francia, sino de mostrar de qué manera se pasó de la rusticidad de esos tiempos al refinamiento de nuestra época». ${ }^{55}$ Es decir, se trata de un parámetro ético-político, según el cual la historia es una historia del «espíritu humano» y sus «progresos» (les progrès de l'esprit humain). ${ }^{56}$ Las críticas a

52 Voltaire, Remarques pour servir de supplément à l'Essai sur les mours [en adelante Remarques], en Essai, t. 2, p. 901.

53 En la entrada «Historre» de la Encyclopédie dedica un apartado a cada uno de los parámetros: «De la certitude de l'histoire» y «De l'utilité de l'histoire».

54 Véase J. Dagen, L'histoire de l'esprit humain dans la pensée française de Fontenelle à Condorcet. Paris: Klincksieck, 1977.

55 Remarques, p. 904.

56 Ibid., p. 906. Para una interpretación diferente, véase J. Dunyach, «L’histoire voltairienne entre progrès et décadence: du Grand Siècle à l'idée de civilisation», Studies on Voltaire 
los relatos absurdos y la mención de los sucesos contrarios a ese progreso se justifican sólo en la medida en que «permiten ilustrar a los hombres» y, de esa manera, rectificar con el tiempo las ideas de las sociedades. ${ }^{57}$

Las críticas de Voltaire a Larcher que van más allá del plano personal responden a este doble criterio. Por un lado, la preocupación por la verdad, el parámetro epistemológico, que lleva a Voltaire a objetar a Larcher su incapacidad para distinguir entre el relato verdadero y el falso, la historia de la fábula. Por otro lado, el parámetro ético-político lo lleva a criticar a Larcher y a los eruditos en general por la inutilidad de sus trabajos de investigación en vistas de una historia del espíritu y sus progresos.

De esta manera confluyen en la obra de Voltaire dos maneras de hacer historia presentes en la época, a saber, la de los eruditos, interesados en el trabajo con los archivos y preocupados por establecer la verdad de los hechos, y la de les philosophes, centrada en aspectos teóricos y generales acerca del curso del «espíritu humano» en el tiempo. ${ }^{58}$ En efecto, Voltaire leía y hacía uso de obras de eruditos como Histoire des empereurs de Louis-Sébastien Lenain de Tillemont o la edición de Nicolas Lenglet Dufresnoy de De mortibus persecutorum de Lactancio. Además, en el momento de preparar una nueva edición de sus obras (edición Khel) revisó una vez más la Philosophie de l'histoire, que desde 1769 se había convertido en la introducción del Essai, e introdujo correcciones acerca de hechos históricos apoyándose nada menos que en el Supplément de Larcher, ya sea modificando expresiones, agregando detalles o directamente abandonando algunas de sus tesis a partir de las refutaciones de Larcher. ${ }^{59}$ Pero, al mismo tiempo, su interés por una «historia del espíritu» lo llevó a descartar sucesos por su falta de «utilidad» y a restar importancia al rigor del método en muchas ocasiones. Así, por ejemplo, dice en Remarques que las historias de Francia, España e Italia de la época no contenían más que «un montón de hechos inútiles» ${ }^{60} \mathrm{y}$ en el prefacio al tomo III de la edición Walther de sus obras, señala que «carece de importancia que el pueblo de Kreuznach haya sido tomado el 21 o el 22 de septiembre de 1688». Inmediatamente se refiere

and the Eighteenth Century, 10 (2006), pp. 133-146.

57 Remarques, pp. 906, 931.

58 Cf. Ch. Grell, L'histoire entre érudition et philosophie, ed. cit., pp. 22 y 23.

59 Voltaire realiza treinta y una modificaciones a partir del Supplément de Larcher. Entre esas modificaciones se encuentran: rectificaciones de referencias a partir de las indicaciones de Larcher (cf. Philosophie de l'histoire, ed. cit., pp. 162, 209); adiciones de detalles tomados del Supplément (cf. Ibid., p. 212); modificaciones de datos cuestionados por Larcher (cf. Ibid., pp. 174, 209); la supresión de tesis, como la de la inexistencia de Nínive y del imperio asirio, que Larcher había refutado en su obra (cf. Ibid., p. 123).

60 Remarques, p. 900. 
con desprecio a «los detalles que no conducen a ningún lugar» ${ }^{61}$ Asimismo, en el prefacio al Essai afirma que el objetivo del trabajo «no es conocer en qué año un príncipe indigno de serlo sucede a otro en una nación bárbara», sino de recordar las acciones de los grandes soberanos «que han hecho a sus pueblos mejores y más felices». ${ }^{62}$ Por otra parte, sostiene que no tiene sentido estudiar la historia de los países que no han cultivado las ciencias y las artes útiles al género humano. En la entrada «CELTES» del Dictionnaire philosophique (1764), señala: «(...) queridos celtas, permitidme deciros, tanto a vosotros como a los hunos, que sociedades como las vuestras que no han cultivado las artes útiles y agradables no merecen nuestro estudio más que los puercos y los asnos que vivían en vuestras tierras». ${ }^{63}$

\section{Conclusiones}

En 1767 Larcher publica una Réponse a la Défense de mon oncle: précédée de la Relation de la mort de l'abbé Bazin; et suivie de l'Apologie de Socrate, traduite du grec de Xénophon (Amsterdam: Changuion). Pero, ¿qué relación existe entre la respuesta de Larcher a Voltaire y la traducción de la Apología de Sócrates? ¿Por qué incluir los dos trabajos en un mismo volumen? El mismo Larcher lo explica en su introducción a la Apología: «Hemos visto la defensa del abate Bazin, ahora veremos la apología de Sócrates. El primero convencido de su ignorancia y de ser un plagiario no responde más que con calumnias atroces e injurias infames. El segundo se comporta de una manera totalmente diferente (...) se defiende con toda la dignidad que permite una conciencia recta que nada tiene que reprocharse. ¡Qué diferencia entre este filósofo antiguo, ese ciudadano sabio y virtuoso y nuestro filósofo moderno!»». ${ }^{64}$ Larcher señala que Voltaire responde a sus críticas sólo a través de «calumnias» e «injurias». Los especialistas no parecen haber interpretado la respuesta de Voltaire de manera diferente. Según John Leigh en La défense de mon oncle no se puede separar el relato histórico de la ficción y las cuestiones biográficas. ${ }^{65}$ Asimismo, José-Michel Moureaux, en la introducción a la edición crítica de

61 Essai, p. 889.

62 Cf. Ibid., p. 195.

63 Voltaire, Dictionnaire philosophique, en Voltaire, Euvres complètes, ed. L. Moland, ed. cit., 1878, t. 18, pp. 107, 108. Lo mismo dice respecto de otros pueblos primitivos en La Russie sous Pierre le Grand: «Todos los pueblos que no han cultivado las artes deben ser condenados al olvido», en Voltaire, Euvres historiques, ed. cit., p. 372.

64 P. Larcher, Réponse a la Défense de mon oncle: précédée de la Relation de la mort de l'abbé Bazin; et suivie de l'Apologie de Socrate, traduite du grec de Xénophon. Amsterdam: Changuion, 1767 , p. 45

65 Cf. J. Leigh, op. cit, p. 107. 
la Défense, preparada por él mismo, cataloga al libro como un «panfleto», un escrito «polémico» y señala que el trabajo sólo cobra sentido si se lo inserta en la coyuntura histórica en la que surge ${ }^{66} \mathrm{El}$ libro es, antes que la defensa de la Philosophie de l'histoire, la defensa de Voltaire mismo, sostiene. ${ }^{67}$

Sin embargo, según se desprende de este trabajo, el libro no es un mero panfleto difamatorio. La respuesta trasciende el plano personal y la coyuntura ideológico-política. Es verdad que las calumnias y las injurias hacia Larcher, así como las marcas de la época, están presentes. Podríamos mencionar, por ejemplo, el enfrentamiento al materialismo ateo, que se trasluce en el capítulo I, dedicado a la providencia, o en el diálogo entre Mandétès y Platón en el capítulo XXI. Podríamos mencionar también la defensa, en ese mismo capítulo, de Belisaire (un trabajo escrito en febrero de ese mismo año por el colaborador de la Encyclopédie Jean-François Marmontel) frente a la crítica del anti-philosophe François-Marie Coger. ${ }^{68}$ Pero la crítica no se agota en el plano de la biografía y la discusión coyuntural, detrás de ese plano encontramos una discusión que se ubica en el campo de la filosofía de la historia y arroja luces acerca de los criterios epistemológicos y filosóficos de «Voltaire historiador». En efecto, Voltaire aplica en la Défense el método diseñado en las Nouvelles considérations sur l'histoire. Es decir, detrás de las calumnias emergen críticas que se apoyan en los criterios establecidos por Voltaire para diferenciar la «historia verdadera» de la que no lo es. Por otra parte, muchos de los temas tratado en la Défense retoman o desarrollan temas abordados en la Philosophie de l'histoire, como el mencionado tema de la prostitución de la damas de Babilonia, tratado en el capítulo II de la Défense y en el XI de la Philosophie; la discusión sobre las contradicciones del Génesis con respecto a la edad de Abraham, presentada en el capítulo VIII de la Défense y en el XVI de la Philosophie, y la reflexión sobre la inverosimilitud de los relatos de Rollin y Bossuet, desarrollada en el capítulo IX de la Défense y en el VI de la Philosophie, entre otros.

En conclusión, La défense de mon oncle es más que un roman o un panfleto. En efecto, es una obra donde salen a la luz los criterios epistemológicos

66 Según Moreaux, Voltaire no se dirige a Larcher en la Défense más que a través de «burlas y sarcasmos», cf. J.-M. Moreaux, «Introduction», ed. cit., pp. 5, 128, 129. Por otra parte, René Pomeau no incluye el trabajo entre las obras que componen su ya mencionada edición crítica de las Euvres historiques de Voltaire.

67 J.-M. Moreaux, «Introduction», ed. cit., p. 127.

68 Jean-François Marmontel, colaborador de la Encyclopédie, publicó Bélisaire en febrero de 1767. En abril el trabajo fue censurado por la Sorbona y Francois-Marie Coger publicó el trabajo crítico Examen du Belisaire de M. Marmontel. Acerca de la polémica en torno a Belisaire, véase J. Renwick, Marmontel, Voltaire and the Bélisaire affair. Oxford: The Voltaire Foundation, 1974. 
de «Voltaire historiador» y se retoman y desarrollan temas tratados en la Philosophie de l'histoire. Traer a la superficie los diferentes estándares utilizados por Voltaire para escribir historia nos ha llevado, por otra parte, a revisar la problemática relación entre los mismos y a mostrar los límites con los que choca su proyecto de acercar la historia a la nueva física, en la medida en que el interés por escribir la historia «del espíritu humano» lo lleva, como se puede observar en III, a no observar en muchas ocasiones los criterios que con ese objetivo había establecido.

\section{REFERENCIAS BIBLIOGRAFICAS}

Borghero, C., «Les philosophes face à l'histoire: quelques discussions sur la connaissance historique aux XVII ${ }^{\mathrm{e}}$ et XVIII ${ }^{\mathrm{e}}$ siècles», en Ch. Grell y J.-M. Dufays (eds.), Pratiques et concepts de l'histoire en Europe, $X V I^{e}-X V I I I^{e}$ siècles. Paris: Presses de l'Université de Paris-Sorbonne, 1990, pp. 73-83.

, Le certezze e la storia: cartesianismo, pirronismo e conoscenza storica. Milan: Franco Angeli, 1983.

Brumfitt, J., Voltaire Historian. Oxford: Oxford University Press, 1958.

CASINI, P., «Progrès de la raison et progrès des sciences chez les encyclopédistes», en L'histoire au dix-huitième siècle. Aix-en-Provence: EDISUD, 1980, pp. 117-133.

CRÉPON, M., «La double philosophie de l'histoire de Voltaire», en B. Binoche y F. Tinland (eds.), Sens du devenir et pensée de l'histoire au temps des Lumières. Seyssel: Champ Vallon, 2000, pp. 77-84.

FERret, O., «Voltaire et les sciences: pour une approche pluridisciplinaire de la question», Revue Voltaire, 8 (2008), pp. 179-195.

Grell, Ch., L'histoire entre érudition et philosophie: étude sur la connaissance historique à l'âge des Lumières. Paris: Presses universitaires de France, 1993.

Guion, B., Du bon usage de l'histoire. Histoire, morale et politique à l'âge classique. Paris: Honoré Champion, 2008.

Larcher, P., Supplément à la Philosophie de l'histoire de feu M. l'Abbé Bazin, nécessaire à ceux qui veulent lire cet ouvrage avec fruit. Amsterdam: Changuion, 1767.

, Réponse à la Défense de mon oncle. Amsterdam: Changuion, 1767.

LeIgh, J., Voltaire. A Sense of History. Oxford: Voltaire Foundation, 2004

MARTIN-HAAG, É., «Les Lumières françaises et l'histoire», en G. Marmasse (ed.), L'histoire. Paris: Vrin, 2010, pp. 53-74.

McGinNIS, R., «L'histoire prostituée. Voltaire contre Larcher, et contre lui-même», The Romanic Review, 88, n 2 (1997), pp. 229-240.

MÉRICAM-Bourdet, M., Voltaire et l'écriture de l'histoire: un enjeu politique. Oxford, Voltaire Foundation: 2012.

Moreaux, J.-M., "Voltaire et Larcher, ou le faux "mazarinier"», Revue d'Histoire littéraire de la France, 74, n 4 (1974), pp. 600-626.

Nonnotte, C.-F., Les erreurs de Voltaire. Besançon: Gauthier Frères, 1818. 
Pierse, S., «Voltaire: Polemical Possibilities of History», en S. Bourgault y R. Sparling (eds.), A Companion to Enlightenment Historiography. Leiden: Brill, 2013, pp. 153-187.

Pomeau, R., Voltaire en son temps. Paris: Fayard, 1995, 2 vols.

Volpilhac-Auger, C., «Voltaire and history», en N. Cronk (ed.), The Cambridge Companion to Voltaire. Cambridge: Cambridge University Press, 2009, pp. 139-150.

Voltaire, Nouvelles considérations sur l'histoire, en Voltaire, Euvres historiques, ed. René Pomeau. Paris: Gallimard, 1957.

, Essai sur les mœurs et l'esprit des nations, ed. René Pomeau. Paris: Garnier, 1964, 2 vols.

, La défense de mon oncle, ed. crítica de José-Michel Moureaux, en CEuvres complètes de Voltaire/Complete Works of Voltaire. Oxford: The Voltaire Foundation, 1984, t. 64.

, Philosophie de l'histoire, ed. crítica John H. Brumfitt, en Euvres complètes de Voltaire/Complete Works of Voltaire. Oxford: The Voltaire Foundation, 1969, vol. 59.

, Correspondance, ed. Theodore Besterman. Paris: Gallimard, 1983, t. 8.

, «Historre», en D. Diderot y J. D’Alembert (eds.), Encyclopédie ou Dictionnaire raisonné des sciences, des arts et des métiers. Paris: Briasson/David/Le Breton/ Durand, 1765, vol. 8, pp. 220-230.

Adrián Ratto es doctor en Filosofía por la Universidad de Buenos Aires. Actualmente es becario postdoctoral del CONICET y se desempeña como docente en la Facultad de Filosofía y Letras (Universidad de Buenos Aires).

Publicaciones recientes:

"Utopie, progrès et décadence dans la philosophie de l'histoire de Voltaire", Revue Voltaire, 2012.

"Soledad y filosofía. Las críticas de Diderot a Rousseau en el Essai sur les règnes de Claude et de Néron", Revista de Filosofía (en prensa).

Lineas de investigación:

Ha participado en diferentes proyectos de investigación ligados a la filosofía de la historia y la filosofía moderna. Sus trabajos de investigación giran en torno a la filosofía francesa del siglo XVIII.

Dirección postal:

Av. Luis María Campos 381, $3^{\circ}$ "A”, Ciudad Autónoma de Buenos Aires, Argentina. CP: 1426.

E-mail:ga_ratto@yahoo.com.ar 